\title{
Point of view: The professional psychiatrist
}

\author{
R Hanwella, C Suraweera
}

\section{Abstract}

A professional has had prolonged specialised training in an abstract body of knowledge and or service orientation. As professionals, there are duties and responsibilities placed on us towards those we serve, our colleagues and ourselves.

Key words: professional, psychiatrist

SL J Psychiatry 2017; 8(2): 31-32
Who is a professional? A professional has had a prolonged specialised training in an abstract body of knowledge or service orientation. Society recognises professionals only among a selected number of occupations. A professional is also legally recognized by some form of licensing, is relatively free of lay evaluation and control, yet should abide to norms of practice enforced by that profession. The members identify with the profession more strongly compared to other occupations. The profession is likely to be a terminal occupation and members are likely to remain in that profession for their lifetime.

A psychiatrist too is a professional. As psychiatrists, let us consider the do's and don'ts which may be important to follow, in our professional lives:

1. Possess a wide range of skills and knowledge relevant to the profession. Professionals never stop learning. Learning does not stop after the MD or Board Certification. With time knowledge becomes out-dated. With time, all of us will need to master new skills or therapies that were not available or taught during the time of our formal training. Psychiatrists are duty-bound to master these skills and acquire knowledge in order to provide the best care for patients. Making use of learning opportunities, attending continuing professional development courses regularly and reading relevant subject matter daily are important in this regard.

2. Engage in evidence-based practice. Sometimes we may have developed certain beliefs and practices, which over time has been shown to be out-dated or wrong. Yet we may hold on to these beliefs and manage our patients accordingly. This would be detrimental to patient wellbeing. This may happen insidiously to all of us, as professionals, and we may not even realize it. Constantly examining our own practice, updating ourselves, and discussions with our colleagues and peer review, would help guard against this fallacy.

3. Have a knowledge of psychotherapy and be able to recommend or use it effectively. Psychotherapy is an important component of psychiatric treatment. Yet this aspect may not have been emphasised in every training programme. In a busy practice, it maybe easier to opt for pharmacotherapy as the treatment option. However, there are many psychiatric disorders where pharmacotherapy is not the best option, or where combining with psychotherapy is the ideal. Certain patients may also be reluctant to take medication. As specialists in psychiatry, we should be sufficiently competent to offer this option, which would set us apart from the non-specialist mental health practitioner.

4. Practice rational pharmacotherapy. Rational pharmacotherapy focuses on prescribing appropriate medications in appropriate doses. Different conditions and different patients need different combinations of medication. We should use our expertise to practice rational multidrug therapy when necessary, but not irrational poly-pharmacy.

5. Dress well. Patients have a certain expectation of dress and behaviour from a doctor. When doctors dress in a slovenly manner it may make patients uncomfortable and damage the doctor-patient relationship. It is our responsibility to dress and comport ourselves in a manner befitting our profession and responsibilities.

6. Know the limits. None of us, as professionals, are equally expert in every aspect of our speciality; while we may have a special interest in a particular area, our experience may be limited in other areas. In such situations, it is best to refer on to a colleague who can manage the patient better - either within the field of psychiatry, or within other specialities. 
7. Be respectful of a patient's culture or religious beliefs. Psychiatrists should never attempt to force their personal, religious or political views on a patient. If such beliefs and practices are seriously negatively impacting on the patient's mental health, we may discuss them, but in a tactful and courteous manner. Our motive should be to overcome barriers necessary for optimum management of the patient's problem, rather than religious or political conversion. We need to be sensitive to the contrasting socio-cultural and educational attitudes or beliefs that may be held by our patients; and these outlooks - which may or may not differ from our own belief systems - should not inappropriately colour our diagnostic discretions.

8. Don't break the confidentiality. This is important for all doctors, but more so for psychiatrists. A psychiatrist is privy to information relating to the most confidential areas of a patient's life; and it is our duty, as psychiatrists, to maintain this confidentiality and trust, that our patients place in us.

9. Maintain good health. In order to deliver highest level of care to our patients we should be in good health. Therefore it is our responsibility, not only to our families, and ourselves, but to our patients as well, to maintain good physical and mental wellbeing. Engaging in regular exercises and activities such as creative hobbies may help us to remain healthy.

10. Be kind to self. We will make mistakes and fall short of the high ideals we expect from ourselves. This happens to all of us, and it is not necessarily a sign of poor commitment or lack of integrity. A professional should be able to acknowledge mistakes, learn from them and take steps to prevent their recurrence. We should not punish ourselves by ruminating. One could share vulnerabilities with a trusted colleague but never with patients. If necessary we could say sorry gracefully, and move on.

\section{Conflict of interest}

None declared

R Hanwella, C Suraweera, Department of Psychiatry, Faculty of Medicine, Colombo

Corresponding author: C Suraweera

Email: dr.chathurie@gmail.com

iD http://orcid.org/0000-0002-7542-5068 